Décadrages Décadrages

cinéma, à travers champs Cinéma, à travers champs

1-2 | 2003

Le hors-champ

\title{
Louis Seguin et la question du hors-champ : une cartographie de l'espace du cinéma
}

\section{Leo Ramseyer}

\section{(2) OpenEdition}

1 Journals

Édition électronique

URL : http://journals.openedition.org/decadrages/590

DOI : $10.4000 /$ decadrages. 590

ISSN : 2297-5977

Éditeur

Association Décadrages

Édition imprimée

Date de publication : 1 octobre 2003

Pagination : 110-120

ISSN : 2235-7823

\section{Référence électronique}




\title{
Louis Seguin et la question du hors-champ: une cartographie de l'espace du cinéma
}

\author{
par Leo Ramseyer
}

1 Louis Seguin, L'espace du cinéma (Horschamp, hors d'œuvre, hors-jeu), Ombres, Toulouse, 1999.

2 André Bazin, Qu'est-ce que le cinéma?, Cerf, Paris, 1999.
La problématique du hors-champ est relativement récurrente dans l'histoire des théories du cinéma. Louis Seguin est le dernier à s'être attelé à cette question dans son livre publié en 1999, L'espace du cinéma, (hors-champ, hors-d'œuvre, hors jeu) $\mathbf{1}$. L'objet du présent article consistera à discuter, en regard des propositions qui les précèdent, les thèses de Seguin, sa cartographie de l'espace au cinéma. Ses réflexions seront donc confrontées aux travaux d'André Bazin, de Pascal Bonitzer et de Gilles Deleuze portant sur le hors-champ. Cette perspective qui survole des périodes très différentes doit faire face à une pluralité de positionnements qu'il est délicat de démêler: les auteurs se situent tantôt dans un discours ontologique, politique ou historique, tantôt à cheval sur les trois. Chacun de ces différents plans détermine un certain type de réflexion, de telle manière qu'il faut parfois une grande souplesse pour les faire se répondre.

Louis Seguin élabore sa théorie avec comme butoir celle d'André Bazin qu'il considère comme la pierre d'achoppement de l'histoire des théories de l'espace au cinéma. Il se focalise principalement sur trois articles: "Montage interdit»(1953-57), «Peinture et cinéma» et "Ontologie de l'image photographique» (1945) 2. Dans une volonté de recommencer à zéro, il commence par récuser deux idées fondamentales pour Bazin:

1. Bazin établit une opposition ontologique entre le cadre qui entoure la peinture et celui qui détermine les limites de l'image cinématographique. C'est la célèbre dichotomie entre le cadre comme zone d'orientation du regard qui, en séparant le «microcosme pictural» du «macrocosme 
naturel ", dirige le parcours de l'œil à l'intérieur du tableau (mouvement centripète), et l'écran de cinéma comme cache, comme fenêtre s'ouvrant sur un espace qui se poursuit infiniment (mouvement centrifuge).

Cette opposition se trouve ébranlée par Seguin lorsqu'il montre que le cadre n'est spécifique qu'à la peinture de chevalet. La fresque ou les polyptyques par exemple ne sont pas soumis à cette règle du cadre pictural que Bazin veut ontologique. Les frontières de l'espace sont soit évanescentes, soit démultipliées et fracturées. Ainsi, l'opposition que construit Bazin devient moins pertinente si, comme Seguin, on prend conscience de la vision réductrice de l'histoire de l'art qu'elle met en œuvre. Aujourd'hui, il faudrait également prendre en compte, à la suite de Bonitzer, des peintres contemporains comme les hyperréalistes, qui, justement, mettent en péril cette fonction du cadre 3 . Toutefois, on ne peut bien sûr pas reprocher à Bazin de n'avoir pas tenu compte de développements de la peinture qui lui sont postérieurs. Ce point témoigne de la complexité d'un discours qui se prétend ontologique.

2. Selon Bazin, l'image cinématographique aurait à voir avec la relique ou, plus précisément, elle serait une image non-faite de la main de l'homme, une image purement indicielle: «Pour la première fois, une image du monde extérieur se forme sans intervention créatrice de l'homme»4. L'image cinématographique est donc, selon Bazin, essentiellement objective. Elle libérerait la peinture de sa volonté de produire une image rationnelle du monde et lui permettrait de se détacher de ses velléités réalistes et de la perspective. La peinture recouvrerait ainsi la liberté, le paradis de l'art médiéval.

Seguin montre, avec l'apport d'Erwin Panofsky, que ces catégories ne sont pas pertinentes et qu'elles reposent sur une connaissance lacunaire de la peinture. La perspective médiévale n'a rien de moins rationnel que celle mise au point à la Renaissance. Selon Seguin, cette position est une "bévue» théorique et, de manière plus générale, il reproche à Bazin la fragilité des fondations de son édifice théorique, son caractère "marécageux". On sent chez lui une volonté de "tuer le père» pour pouvoir asseoir ses propres théories. Le caractère idéaliste de la théorie de Bazin n'a pas attendu Seguin pour être sérieusement remis en cause ${ }^{5}$. Le cinéma n'est donc pas cette épiphanie du réel, il est pure représentation, au même titre que la peinture.

Les frontières entre peinture et cinéma ne sont donc plus aussi tranchées: les distinctions telles que centrifuge / centripète ou "fait-dela-main-de-l'homme» / geste divin sont détruites dans leur fondation même et l'on entre dans une théorie qui n'a plus besoin de promouvoir
3 Pascal Bonitzer, Le champ aveugle. Essais sur le cinéma, Gallimard, 1982, Paris, p. 115.

4 Qu'est-ce que le cinéma?, op. cit., p. 13.

5 Voir entre autres la polémique autour des "effets produits par l'appareil de base" dans les années 70; entre ceux qui considèrent le dispositif comme étant purement idéologique (Jean-Louis Baudry, Jean-Louis Comolli,...) et ceux qui voient la caméra comme idéologiquement neutre, objective (Jean-Patrick Lebel, Jean Mitry,...). 
6 Qu'est-ce que le cinéma?, op. cit., p. 160.

7 Pascal Bonitzer, "Hors-champ (un espace en défaut)", in Cahiers du cinéma, n 234-235, décembre 1971-janvier/février 1972. les spécificités du cinéma par rapport aux autres arts. Pour Seguin, l'espace du cinéma, à l'instar de celui de la peinture, ne se construit que sur l'écran, il est centripète. Le cinéma n'est pas un jeu de cache-cache, tout ce qui doit être montré l'est sur l'écran. Le cinéma ne diffère rien, il ne se déporte pas dans un espace infini qui serait le hors-champ. "Au cinéma, tout est sur l'écran et nulle part ailleurs. Il n'y a rien devant lui que ce qui force l'espace du cadre» (Seguin, op. cit., p. 47). On entre ici dans le vif de la conception de «l'espace du cinéma» que propose Louis Seguin.

Seguin veut en finir avec le mythe de «la robe sans couture de la réalité» qui veut que champ et hors-champ coexistent en toute continuité et homogénéité. Ce système est ainsi synthétisé par Bazin :

"Quand un personnage sort du champ de la caméra, nous admettons qu'il échappe au champ visuel, mais il continue d'exister, identique à lui-même, en un autre point du décor, qui nous est caché» $\boldsymbol{6}$.

Cette idée d'un espace qui se prolonge infiniment au-delà du cadre, d'où les personnages entrent et sortent et dans lequel ils continuent d'exister en d'autres endroits invisibles, est pour Seguin une défaillance de la pensée: «Le hors-champ appartient au bon sens. Il est, comme lui, la chose du monde la mieux partagée» (op. cit., p. 117). Il serait une sorte de tranquillisant théorique destiné à se protéger de l'étrangeté de l'écran. Le hors-champ est un leurre conçu pour détourner l'attention. Pascal Bonitzer relève aussi cette fonction de détournement du hors-champ en tant que construction idéologique et la dénonce dans une approche politique 7 . Selon lui, le hors-champ est utilisé par l'idéologie dominante ("petite-bourgeoise») pour court-circuiter la matérialité du cinéma, c'est-à-dire tout ce qui pourrait entraver la consommation jouissive des films. C'est un leurre qui masque ce que le cinéma veut vraiment cacher, son "espace de production" (espace de la caméra, du preneur de son, etc.). Cet appendice théorique qu'est le hors-champ entraîne le cinéma à se nier en tant que médium:

"Dans le système de cet espace, on a donc affaire comme aux deux faces d'une même opération 1. à un geste d'exclusion radical (forclusion de la matérialité de la scène filmique) 2. à l'investissement de l'espace d'exclusion d'une réalité fictive, continuant l'espace du champ.» (Ibid.).

La matérialité filmique est rejetée hors du cadre (en fait juste à côté, tout contre le cadre) et la béance ainsi creusée se voit maquillée par le horschamp qui laisse ainsi le spectateur dans la méconnaissance du processus cinématographique. Cette méconnaissance est la caractéristique, selon Bonitzer, d'un cinéma qui corroborerait le discours dominant petit-bourgeois. 
Seguin et Bonitzer dénoncent ainsi tous deux le hors-champ comme leurre. Pour l'un, c'est «l'anesthésique de la théorie, ce qui permet de se tranquilliser et de s'endormir, de se retirer au calme, au large, loin des tracas, des inquiétudes et des étrangetés de l'écran" (op. cit., p. 117), pour l'autre c'est un moyen de pression idéologique. On peut remarquer encore qu'entre le "bon sens» et l'idéologie "petite-bourgeoise», il n'y a qu'un pas. Reste posée la question de ce qui est découvert lorsque l'on dénonce et déchire "ce bâtard surdoué» (Seguin, op. cit., p. 97) qu'est le hors-champ.

Pour Seguin, l'espace du cinéma est clos, circonscrit. Il se termine sur les bords de l'écran. L'espace, au fur et à mesure qu'il se rapproche du cadre, se distend. Il subit une distorsion et devient flou. Il se replie sur lui-même dans une "physique implosive» $\mathbf{8}$ pour ne s'intéresser qu'à son milieu. Le cinéma n'est pas un jeu de "cache-cache». L'écran détermine le cadre dans lequel tout doit s'inscrire et au-delà duquel plus rien n’a le droit d'exister, plus rien ne doit être déporté. Le cinéma n'est pas un «spectacle du monde» (Seguin, op. cit., p. 60), c'est le spectacle d'un univers propre et cohérent qui s'inscrit entièrement dans le rectangle délimité par le cadre.

Cet espace centripète s'organise donc entre ces limites floues et distendues qui cernent l'écran. Seguin utilise le modèle de la marge des manuscrits romans du XIII ${ }^{\mathrm{e}}$ siècle pour expliquer le statut et le fonctionnement de ce pourtour trouble. Ces textes s'entourent effectivement d'une sorte de "forêt primitive, un théâtre de l'inextricable hanté par les gnomes et les dragons» (op. cit., p. 57). Les bords de la page se peuplent d'une végétation tentaculaire et de créatures étranges. Ces marges, sorte de négatif du texte, sont du ressort de l'irrationnel. C'est depuis ces marges, en périphérie du texte, que le blasphème, le sexe et la mort viennent hanter le corps même des écrits les plus respectables. Ces marges sont le lieu indéterminé, irréel et carnavalesque qui «cerne et ouvre l'abîme de la figure» (op. cit., p. 58). Elles ne sont pas foncièrement constitutives du texte, mais elles en sont pourtant inséparables et exercent une influence sur lui. Le cinéma fonctionnerait de la même manière. Plus l'espace se rapproche du cadre (entendu comme limite), plus il se contorsionne, se cambre et se replie sur lui-même. C'est de cette courbure, de cette zone indistincte que les personnages naissent et meurent dans un incessant mouvement de résurrection. Les personnages, les décors, la nature s'enfoncent et émergent de ce néant "comme des diables de leurs boîtes» (op. cit., p. 95). Lorsque ce qui est vu disparaît, il n'est pas mis en réserve dans les limbes d'un quelconque horschamp, il est privé d'existence, de présence, il meurt, il s'engouffre dans l'épaisseur insaisissable de cette zone frontière.
8 Louis Seguin, "Aux distraitement désespérés que nous sommes..." (Sur les films de JeanMarie Straub et Danièle Huillet.), Ombres, Toulouse, 1991, p. 22. 
9 Livio Belloi (Poétique du hors-champ, Revue belge du cinéma, $\left.\mathrm{n}^{\circ} 31,1991\right)$ organise toute son étude autour des concepts de dedans et de dehors, et établit les relations qu'ils entretiennent (trois régimes: l'empreinte, l'adresse et le passage) au fil de périodes rigidement tranchées dans l'histoire. II dégage, entre autres, le cinéma du dedans qui s'organise sans aucun extérieur, qui se présente, autonome, comme le cube théâtral. La saute temporelle qui permet à la magie d'advenir serait le dehors, le horschamp inscrit au sein de l'image.

10 Edgar Morin, Le cinéma ou l'homme imagi naire, Minuit, Paris, 1978 [1956], p. 118.

11 L'homme ordinaire du cinéma, Gallimard, Paris, 1980.

12 Gilles Deleuze, Cinéma 2. L'image-temps, Minuit, Paris, 1985, p. 343.

13 Gilles Deleuze, Pourparlers, Minuit, Paris, 1990 , p. 204.
Il y a une part de mystère et de magie dans le fonctionnement de ce sas qui rappelle les tours de prestidigitation de Méliès et son esthétique de la «saute»9. Peut-être aussi que cet aspect irrationnel de l'approche de Seguin pourrait trouver une résonance dans les travaux d'Edgar Morin. L’analyse de Seguin pourrait être comprise comme une topographie de la "perception magique» que théorise Morin. Ce serait une manière de nouer (de ramener sur un même plan) l'image cinématographique et sa perception spectatorielle magique pour en dessiner une cartographie. Seguin ne ferait qu'explorer cette "magie latente de l'image» $\mathbf{1 0}$ et dresser un carnet de bord des nombreux rapports que l'image entretient avec le rêve, la névrose, la régression, le merveilleux, la folie. Il rapatrie dans le champ théorique les mécanismes de réception dégagés par Morin. Seguin joue la perception magique contre Bazin: «la trame des rêves n'est pas faite de la même étoffe que la robe sans couture de la réalité; elle se déchire, se coupe, se recoud et s'ourle» (op.cit., p. 107). Cette position, à mi-chemin entre une sorte de "psycho-mécanique» de la réception et une approche plus esthétique de l'image cinématographique rappelle celle de Gilles Deleuze qui, avec ses «automates psychologiques et spirituels» ainsi que ses circuits tendus entre le cerveau du spectateur et l'écran, retrouve certaines intuitions de Morin ainsi que les recherches de Jean-Louis Schefer11. Plus généralement, c'est peut-être une volonté de définir un nouveau spectateur qui ne serait plus strictement assimilé au «sujet psychologique». C'est l'idée que le statut du spectateur (son expérience) ne serait pas épuisé par une lecture psychanalytique, dont les outils théoriques ont été sérieusement remis en cause (par Deleuze notamment), et qu'il faudrait en appeler aux modèles du «mannequin", de l'automate, de la machine ou de «l'homme mécanique et sans naissance»12. On voit ici ce désir, partagé par Seguin, d'éviter l'utilisation trop mécanique des théories psychanalytiques dans l'étude conjointe du statut du spectateur et du film. Le véritable enjeu est de percer l'organisation des circuits qui sont inventés et tracés par le cinéma dans la matière indifférenciée qu'est le cerveau, étant donné que ces chemins ne sont pas préexistants. Deleuze synthétise ainsi cette perspective: «Ce qui m’a intéressé dans le cinéma, c'est que l'écran puisse y être comme un cerveau.»13

Si le "bon sens» (ou l'idéologie petite-bourgeoise) a enfanté le horschamp, c'est peut-être pour se protéger de cet irrationnel, se distancier de cette magie qui, selon Morin, est constitutive du cinéma. C'est cela le tranquillisant: la volonté de ne pas voir tout ce qui hante l'écran et qui représente un véritable danger. C'est aussi ce que découvre Bonitzer lorsque, le voile du hors-champ levé, il est confronté aux trous et aux 
béances qui ne sont plus masqués. Qu'est-ce qui fait retour par ces trous? "Les fantômes du regard et de la voix qui hantent et hallucinent les bords de l'image»14. Lui aussi se retrouve face à cet espace indéterminé camouflé par le hors-champ, mais c'est avec le concept du «stade du miroir» élaboré par Jacques Lacan qu'il appréhende cette zone floue jusque-là cachée15. Le hors-champ serait, selon Bonitzer un «lieu d'incertitude et d'angoisse» car, au même titre que le corps de l'enfant lorsqu'il n'est plus reflété par un miroir se disloque et se morcelle, il est impossible de savoir si les personnages, lorsqu'ils franchissent la limite du cadre, n'explosent et ne se désagrègent pas non plus. Au-delà des limites du cadre règne donc un monde hostile et inconnu, hanté par le morcellement des corps, la mort et des fantômes. Bonitzer ne va néanmoins pas aussi loin que Seguin et ne se détache jamais vraiment d'une certaine idée de hors-champ. Lorsqu'il parle du son, il propose même une définition très conventionnelle du hors-champ comme étant homogène au champ et il rejoint (ou a été rejoint par) les distinctions traditionnelles entre son hors-champ et son off que Michel Chion explorera ultérieurement16 et que Deleuze utilisera pour composer son image-mouvement ${ }^{\mathbf{1 7}}$. Pourtant, lorsqu'il parle de l'anamorphose comme «arrière-monde de la perspective» $\mathbf{1 8}$, il est difficile de ne pas faire un rapprochement avec cette courbure, cette déformation géométrique que subit l'espace lorsqu'il se rapproche de ses limites. Cette distorsion théorisée par Seguin serait l'arrière-monde de l'espace filmique, son au-delà non déporté, non différé mais ancré dans la chair même de l'image. L'anamorphose ne se révèle pleinement que lorsqu'elle est reconstituée par le spectateur. On retrouve ici une position théorique à cheval sur une ontologie de l'image cinématographique et une approche presque géographique de la réception du cinéma. Cet au-delà radical ne réside pas dans la continuité du monde extérieur (le hors-champ), il est «un dehors plus lointain»19, absolu, qui hante le cœur même de l'image. Deleuze et Bonitzer anticipent ici cet «ourlet» évoqué par Seguin, cette idée que l'ailleurs n'est pas rejeté au-delà du cadre mais qu'il est un repli de l'image et qu'on ne peut pas l'en dégager.

Le cinéma selon Seguin ne s'appuie pas sur la réalité du monde et l'espace filmique n'a rien à voir avec celui du réel. Il construit un univers propre qu'il encastre entièrement dans le cadre et qu'il force à ne se déployer nulle part ailleurs. Ce monde est conçu comme la volonté d'un auteur qui construit avec chaque mouvement de caméra, chaque plan un espace idéal et chaotique qui ne doit rien à la réalité. "L'extérieur est rejeté vers la nostalgie de la transparence», ce qui importe, c'est cet enfermement, «cette clôture où le lieu et le récit se redoublent» $\mathbf{2 0}$, ce
14 Pascal Bonitzer, Le champ aveugle, Essais sur le cinéma, op. cit., p. 107.

15 Pascal Bonitzer, Le regard et la voix. Essais sur le cinéma, UGE (10/18), 1976, dans l'article "Des hors-champs".

16 Michel Chion, Le son au cinéma, Editions de l'Etoile, Paris, 1994.

$\mathbf{1 7}$ Le regard et la voix, dans l'article "Les silences de la voix", p. 31.

18 Pascal Bonitzer, Peinture et cinéma. Décadrages, Editions de l'Etoile, Paris, 1985, p. 58.

19 L'image-temps, op. cit., p. 363.

20 "Aux distraitement désespérés que nous sommes...", op. cit., p. 35. 
$21 / d .$, p. 132

22 L'image-temps, op. cit., p. 306. Plus loin (p. 361): "C'était le double aspect de l'imagemouvement, définissant le hors-champ: d'une part elle communiquait avec un extérieur, d'autre part elle exprimait un tout qui change. Le mouvement dans son prolongement était la donnée immédiate, et le tout qui change, c'està-dire le temps, était la représentation indirecte ou médiate".

23 Même si Deleuze ne le dit jamais explicitement, l'image-temps recueille toutes ses faveurs car elle est en quelque sorte la seule qui réalise les possibilités immanentes à l'esthétique du cinéma.

24 ld., p. 362. caractère claustrophobique et névrosé de l'espace au cinéma que Seguin décrit comme suit:

«La représentation s'enferme derrière sa frontière. [...] Elle se replie à l'intérieur de ses limites et jouit de sa schizophrénie jusqu'à accepter l'issue catatonique de son repli» $\mathbf{2 1}$.

Le cinéma se barricade et s'entoure d'un champ de mine. Quiconque voudra s'aventurer au-delà du cadre et se mêler ainsi aux affaires du film se verra puni. Il n'y a pas d'intermédiaire entre la fiction et le réel, pas de limbe ou de réserve qui pourrait servir de refuge. "Inutile de regarder le film si vous ne suivez pas les règles, si vous n'admettez pas que vous vous taperez la tête contre les murs si vous voulez en (sa)voir trop " (Seguin, op. cit., p. 107). L'espace, la fiction et le spectateur se redoublent en une seule féerie irrationnelle. C'est presque un mot d'ordre épistémologique : il faut concentrer tous les niveaux d'analyse sur un seul et même plan.

Si Seguin récuse l'existence même du hors-champ, Deleuze, dans un premier temps, lui concède une existence, lorsqu'il parle de l'imagemouvement. Celui-ci est peuplé de cette présence spécifique au sonore, il est séparé en un hors-champ relatif et un hors-champ absolu, l'à-côté et l'ailleurs. "Tantôt le hors-champ renvoie à un espace visuel, en droit, qui prolonge naturellement l'espace vu dans l'image, [...], tantôt, au contraire, le hors-champ témoigne d'une puissance d'une autre nature, excédant tout espace»22. Il rejoint les analyses de Bonitzer ou de Chion sur la répartition du son en in, hors-champ, off ou encore off-off. Tout cela présuppose une dynamique extensive où le champ est prolongé par un hors-champ qui se construit en fonction de l'image visuelle. Le sonore est subordonné au visuel et l'espace s'articule de manière dialectique entre le champ et le hors-champ. On est ici en terrain connu et sécurisé. Mais cette approche n'est valide selon Deleuze que pour l'image-mouvement.

Il en va autrement de l'image-temps ${ }^{23}$. "Il n'y a plus lieu de parler d'un prolongement réel ou possible capable de constituer un monde extérieur: nous avons cessé d'y croire, et l'image est coupée du monde extérieur»24. Deleuze rejoint donc le camp de ceux qui ne veulent plus de cet appendice rationnel qu'est le hors-champ. L'image perd ses "coordonnées euclidiennes» et sombre dans l'indéterminé. En fait, c'est avec l'autonomisation de la bande sonore, sa dissociation d'avec l'image visuelle, la mise à mort de la redondance qui caractérisait leur relation qu'elle devient une «image sonore", disjointe de "l'image visuelle». Le son n'est plus une composante de l'image visuelle, il acquiert désormais son propre cadrage, indépendant de celui de l'image.

«Il n'y a donc plus de hors-champ, pas plus que de sons off pour le peupler. [...] Maintenant l'image visuelle a renoncé à son extériorité, 
elle s'est coupée du monde et a conquis son envers, elle s'est rendue libre de ce qui dépendait d'elle. Parallèlement, l'image sonore a secoué sa propre dépendance, elle est devenue autonome, a conquis son cadrage. A l'extériorité de l'image visuelle en tant que seule cadrée (hors-champ) s'est substitué l'interstice entre deux cadrages, le visuel et le sonore, la coupure irrationnelle entre deux images, la visuelle et la sonore» $\mathbf{2 5}$.

Ainsi, dans l'entrelacement de ces deux images se construit la véritable image audiovisuelle travaillée non plus par un hors-champ qui est mort, mais par cette coupure irrationnelle qui parcourt les limites des deux cadrages. L'organisation classique et logique du champ / hors-champ est remplacée chez Deleuze par cette coupure irrationnelle. Les thèses de Seguin, même si elle ne se superposent pas à celle de Deleuze, en offrent une certaine résonance.

\section{Un espace périphérique: le son}

C'est entre autres sur la question du son - centrale chez Deleuze et relativement peu abordée par Seguin - que l'on trouve des dissonances. Seguin fait un sort un peu trop lapidaire au son: "Les sirènes que l'on entend périodiquement dans Fenêtre sur cour ne sont pas les rumeurs d'un lointain quelconque. Elles sont là, elles aussi, dans la cour et sur l'écran» (op. cit., p. 79). Et plus loin: "Dans un champ-contrechamp, l'interlocuteur est toujours là parce qu'il se reflète sur le visage, dans le regard voire dans les yeux de l'autre mais aussi parce que, même si on ne le voit pas parler, on entend sa voix, non pas par-derrière, de l'autre côté des fauteuils, mais devant, sur la toile blanche où le film est projeté. Il est présent parce que sa voix passe par les petits trous de l'écran» (op.cit., p. 113-114). Il y a chez Seguin une identité entre la localisation diégétique du son et le dispositif qui le restitue dans la salle. Cette confusion l'amène à fustiger, à la suite des Straub, la stéréophonie, comme si la source du son était aussi localisable que la lumière projetée sur l'écran et que ce dernier absorbait dans son centre toute la bande sonore. Le sonore n'a donc chez Seguin pas d'autonomie propre, il est comme entièrement généré par l'image. Cette vision est un peu réductrice et renouerait presque avec les théories, pourtant réfutées, qui organisent différents types de hors-champ en fonction du rapport qu'entretiennent entre eux la bande sonore et le champ visuel. Le son subit la domination de l'image. C'est nier que les sirènes évoquées creusent des galeries, tracent des circuits dans l'espace mental représenté, dans la matière indéterminée du cerveau.

Lorsqu'il appréhende le son, on dirait que Seguin perd de vue le spectateur. Le son ne prolonge pas l'espace sous forme de hors-champ
25 ld., p. 328. 
26 Noël Burch, Une praxis du cinéma, Gallimard, 1986, p. 39-58.

27 ld., p. 28 et 29. certes, mais il lui ajoute des bras tentaculaires. Il dessine des couloirs labyrinthiques et creuse des siphons caverneux. L'espace perçu déborde le cadre sans pour autant en appeler à une continuité. L'espace du cinéma se décolle de l'écran et vient s'incruster, polymorphe (ni rectangulaire ni plat), dans notre cerveau. Cet espace mental est clos. Il ne se poursuit pas infiniment. Il s'arrête là où l'auteur cesse de créer des circuits, là où la représentation se termine et c'est face à cette limite, non à celle du cadre, que l'espace se recroqueville sur lui-même et qu'il se fait envahir par cette étrange population de monstres, de fantômes et de gnomes. Franchir cette frontière qui ne se superpose pas aux limites du cadre, c'est se retrouver dans les coulisses, nez à nez avec le preneur de son, la caméra, l'acteur ou celui qui l'a doublé en français. Seguin lâche le spectateur (sa perception) là où Deleuze concentre en une seule membrane sensible le son, le cerveau et l'écran. Deleuze mène à son terme une volonté de désacralisation que l'on trouve également chez Seguin en remplaçant le lien classique et bazinien oil-nature par cette nouvelle proposition cerveau-écran.

Cette négligence de la question du son est révélatrice de la démarche générale de Seguin. Il considère que l'espace a toujours été subordonné au temps et le premier chapitre de L'espace du cinéma est consacré à illustrer cette prédominance dans l'histoire de la philosophie avec trois exemples: Descartes, Spinoza et Kant. Par extension, il voit dans les discours portés sur l'espace au cinéma le même rapport de force. La peinture n'est pas un art du temps. Le cinéma occuperait cette place, à côté de la pensée et de l'esprit. L'espace siège lui entre le corps et la matière. Seguin veut renverser cette hiérarchie et cette volonté sous-tend toute son analyse. Le rapatriement de l'espace filmique à l'intérieur du cadre et sa superposition avec l'espace pictural est un mouvement qui tend à libérer cet espace de la tutelle du temps. C'est Noël Burch qui fait subir à l'espace ses pires humiliations, avec le devenir champ du hors-champ, cette manière de construire l'espace dans la succession où finalement Burch ne parle plus que de temps ${ }^{26}$. L'espace chez Burch n'est défini qu'en relation avec l'entre-plan, il est toujours à chercher dans le plan suivant, derrière l'image, dans ce sixième segment du hors-champ qui se cache derrière l'horizon. L'espace se dissout dans le temps et Burch se voit contraint, pour forger ses "types de rapport entre l'espace d'un plan A et celui d'un plan B», d'analyser tout plan dans la succession de tout le film, avec des catégories telles que "continuité», "discontinuité» et «manifestement proche» $\mathbf{2 7}$.

Livio Belloi reprend à son compte, peut-être malgré lui, cette manière de dissoudre complètement l'espace dans le temps lorsqu'il 
détourne le concept de l'idée hégélienne pour, en fait, le superposer assez mécaniquement aux théories de Burch 28.

Revenons à Seguin qui note: "Le hors-champ est le complice du temps. Il le fait passer par la porte de service. Il le sort de l'espace pour lui permettre de se replacer, de reprendre les marques de sa domination" (op. cit., p. 55). C'est en cela que le son pose problème dans la réflexion de Seguin, en ce sens qu'il est comme le messager du temps, son garant ou son métronome. La musique, le continuum sonore se déploie dans la durée et se soumet au "temps réel» qu’il est supposé chronométrer. Dans ce putch auquel s'emploie Seguin, le son fait figure de résistant. Il est le bras droit du temps qui ne se laisse pas soumettre. C'est aussi ici que le travail de Deleuze va dans une autre direction, il ménage, lui, un espace où le temps pourra s'épanouir pleinement. Et ce n'est pas pour autant que Deleuze reconduit la dictature du temps, c'est par l'espace qui se détache du monde, qui perd ses liens ("sensori-moteurs») avec l'extérieur et qui devient quelconque, que peut apparaître une image directe du temps. C'est justement lorsque l'espace ne se soumet plus au temps mécanique et automatique (reconstitué) de l'image-mouvement, qu'il peut faire entrer en son sein (pas par la porte de service) une image pure du temps. On voit donc qu'il n'est pas si intéressant d'inverser mécaniquement les rapports de force si l'on reste dans une dynamique de la tyrannie. L'analyse de Seguin aurait gagné en finesse si elle n'était pas aussi déterminée par la volonté de soumettre et d'humilier à son tour le temps. Seguin s'explique sur ce renversement:

"Cette révolution n'est pas un dîner de gala, même si elle reste formelle. Elle implique un retournement de l'idée que l'on se fait ordinairement du cinématographe. Elle invite à se replier, à soutenir un siège, à se concerter sur un espace qui a été privé de ses alibis, de ses utopies, des sorties que lui ouvraient la maîtrise du temps et la création divine. Le cinéma n'imite pas, contrairement à ce qu'avait pu supposer André Bazin, le geste surhumain, inhumain, du Dieu créateur. Il doit en rester [...] à l'immanence de son spectacle» (op. cit., p. 45-46). Seguin veut en finir avec ce "réalisme théologique du hors-champ»29, symptôme de cet assujettissement de l'espace dans le champ théorique. Le concept de "cartographie» évoqué plus haut et la topographie sont bien les outils d'une étude de l'espace, un travail de géomètre qui n'a pas de compte à rendre au temps. Seguin nous exhorte à parier sur la géographie. La géographie contre l'histoire, l'espace contre le temps. Cette manière de toujours construire son analyse contre, cette volonté de révolution, amène peut-être Seguin à une position trop tranchée pour être nuancée.
28 Revue belge du cinéma, op. cit., p. 42-46. Les trois temps de l'idée dans le système de pensée du mouvement de Hegel seraient répartis ainsi: l'en-soi serait le plan inaugural du film et plus particulièrement toute la virtualité qu'il programme (cette virtualité spécifique au premier plan est primordialement et entièrement contenue dans le hors-champ); lorsque cette virtualité s'actualise (entendre passe du horschamp au champ), elle est être-là. Finalement, chaque fois qu'une virtualité est découverte, matérialisée, elle engendre une nouvelle virtualité, un nouveau germe. Ce mouvement (le pour-soi) se propage en boucle jusqu'au plan final, en-soi qui programme des virtualités impossibles.

29 "Aux distraitement désespérés que nous sommes...", op. cit., p. 115. 
Pourtant cette approche, ces résonances trouvées dans la peinture, ces importations de concepts philosophiques, cette espèce d'épaisseur fantomatique à laquelle il nous confronte et son style trouvent une consistance tout autre que certaines autres théories. La lecture de la synthèse historique de Livio Belloi, par exemple, se métamorphose parfois en celle d'un manuel de marketing contemporain. Il est toujours question de "dynamiser l'espace» et de «l'animer», comme si le cinéma devait prouver sa motivation dans le cadre d'un entretien d'embauche ("Quels sont vos trois points forts? Dynamisme, fusion, continuité.»). L'approche de Seguin, comme celle de Deleuze, insuffle au domaine des théories sur le cinéma un certain renouvellement. C'est qu'ils ne se contentent pas de retourner, de retrousser ou de reconduire les concepts qui forment le fond de commerce de l'histoire de la théorie du cinéma. Ils importent et font concerter des concepts empruntés à d'autres domaines en même temps qu'ils mettent en examen les outils et les «acquis» de la théorie. Ces lectures permettent de sortir du vase clos que forme le corpus traditionnel des études sur le hors-champ. 